\title{
Searching for millicharged particles produced in cosmic-ray air showers
}

\author{
Víctor M. Muñoz, ${ }^{a, *}$ Carlos A. Argüelles ${ }^{b}$ and Kevin J. Kelly ${ }^{c}$ \\ ${ }^{a}$ Instituto de Física Corpuscular, Universidad de Valencia and CSIC, \\ Edificio Institutos Investigacíon, Catedrático José Beltrán 2, 46980 Spain \\ ${ }^{b}$ Department of Physics \& Laboratory for Particle Physics and Cosmology, Harvard University, \\ Cambridge, MA 02138, USA \\ ${ }^{c}$ Theoretical Physics Department, Fermilab, \\ Fermilab, P.O. Box 500, Batavia, IL 60510, USA \\ E-mail: carguelles@fas.harvard.edu, kkelly12@fnal.gov, vicmual@ific.uv.es
}

\begin{abstract}
Particles with small electric charges can be produced abundantly in extensive air-showers, generated from the collisions of cosmic rays with the earth's atmosphere. These millicharged particles can leave their imprints at underground experiments via scattering-off electrons. In this report, we present updated constraints using low energy data from Super-Kamiokande, and the XENON1T experiment. Moreover, we provide new sensitivity projections for millicharged particles in the future JUNO neutrino observatory, and discuss different search strategies including both, singlescattering events, and multiple-energy-deposition traces.
\end{abstract}

$37^{\text {th }}$ International Cosmic Ray Conference (ICRC 2021)

July 12th - 23rd, 2021

Online - Berlin, Germany

\footnotetext{
*Presenter
} 


\section{Introduction}

Historically, cosmic-rays and particle physics have been connected. In fact, two elementary particles of the Standard Model (SM) of particle physics were discoved by studying cosmic-ray signals; the positron in 1932 [1], and the muon in 1936 [2]. In the early times, cloud chambers were used to visualize the passage of ionizing radiation. Since then, the level of sophistication of particle detectors has evolved and modern particle detectors usually consist of layers of subdetectors, designed to search for particular properties and specific types of particles. Today we can rely on different types of detection techniques to search for millicharged particles (MCPs) [3], and if a particle with such feature do actually exist, then it should be produced along with the shower radiation generated in the sky when cosmic-ray impacts the earth.

There are very well motivated reasons to search for MCPs. On one side, millicharged particles allow us to test the principle of charge quantization, which, up to now, seems to be a well stablished observation. In addition to this, as millicharged particles interact feebly with the SM, they can be candidates to explain another well established observation: dark matter (DM). In this last scenario it is not possible that MCPs consititute the entirety of the observed abundance of DM, yet it is still plausible that part of the dark matter be in the form of millicharge particles [4].

For the purpose of this study, we adopt a phenomenological approach and assume that millicharged particles are massive, stable particles, which couples to the photon via

$$
\mathcal{L} \supset \varepsilon e A_{\mu} \bar{\chi} \gamma^{\mu} \chi
$$

where $\chi$ denotes the MCP and $\varepsilon \ll 1$. We remain agnostic as to the origin of such coupling, but we stress that this interaction will imply that millicharged particles arriving to undergroud experiments will produce electron recoil signals, via the interchange of the photon. Here, we will report on the study of such signals, and we will show that large underground detectors with low energy thresholds such as JUNO, are particularly very well suited to search for MCPs, and place stringent limits on the charge fraction $\varepsilon$.

\section{Production and propagation of millicharged particles from air-showers}

An extensive cascade of radiation, ionized species, and hadrons is generated when a cosmic ray hits our atmosphere. Among the particles present in the cascade, pseudoscalar and vector mesons including $\pi^{0}, \eta, \rho, \omega, \phi$ and $J / \Psi$, can decay to MCPs. This will give rise to a flux of millicharged particles, which at the surface of the earth can be estimated by [5]

$$
\frac{d \Phi_{\chi}}{d E_{\chi} d \Omega}=\sum_{\mathfrak{m}} \int d X \int d E_{\mathfrak{m}} \frac{1}{\rho(X) \lambda_{\mathfrak{m}}\left(E_{\mathfrak{m}}\right)} \frac{d \Phi_{\mathfrak{m}}}{d E_{\mathfrak{m}} d \Omega}\left(E_{\mathfrak{m}}, X\right) \frac{d n}{d E_{\chi}}\left(\mathfrak{m} \rightarrow \chi ; E_{\mathfrak{m}}, E_{\chi}\right),
$$

where the sum runs over all the mesons in the shower, $\rho$ is the atmospheric density, $\lambda_{\mathfrak{m}}$ is the decay length of the meson $\mathfrak{m}, \frac{d \Phi_{\mathfrak{m}}}{d E_{\mathfrak{m}} d \Omega}$ is the production rate of the meson at column depth $X$, with energy $E_{\mathfrak{m}}$ at a given zenith angle $\theta$, and $\frac{d n}{d E_{\chi}}$ is the energy distribution of the millicharged particle in the decay of the meson. In figure 1 we show the total flux expected at the surface of the earth, for a $10 \mathrm{MeV}$ millicharged particle, as well as the individual contribution from each one of the mesons produced in the atmospheric shower. The arrival direction is fixed by the cosine of the zenith angle 


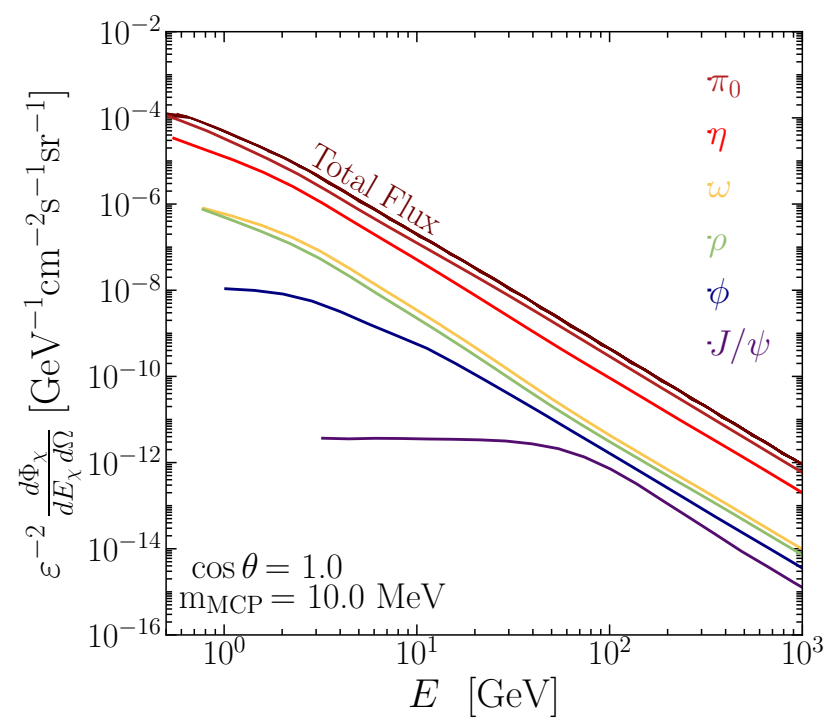

Figure 1: Differencial flux spectrum of millicharged particles produced from different parent mesons in cosmic ray air-showers. The expected MCP flux arriving at the surface of the earth scales as $\varepsilon^{2}$. The arrival direction is fixed, with a zenith angle of $0^{\circ}$. The mass of the millicharged particle is set to $10 \mathrm{MeV}$.

at zero degrees. As can be inferred from this result, the flux of MCPs for higher masses will become increasingly lower, as the contribution from the lightest mesons will be restrictive. The meson production rate were extracted using the MCEq package [6], following the procedure from reference [7].

The MCPs with enough energy will be able to penetrate the earth and propagate underground until they reach the detector. The detection of millicharged particles requires knowledge about charged particle propagation in the medium, as underground experiments uses the Earth's crust to shield from the large background of atmospheric muons. As the MCPs propagates through the earth, it is expected that they lose part of its energy by ionizing the medium and by interacting with nuclei. We assume that energy losses will be mostly independent of the mass for the $\mathrm{MeV}$ regime, and that the mean loss is proportional to squared of the charge fraction $\varepsilon$. Taking this into account, we expect that if the MCP reaches the surface of the earth with an initial energy $E_{i}$, then the average penetration depth $R$ until it reaches the detector with an energy $E_{f}$, will be given by

$$
R=\frac{1}{\varepsilon^{2} b} \ln \left(\frac{1+\frac{a}{b} E_{i}}{1+\frac{a}{b} E_{f}}\right)
$$

where $a$ and $b$ are the energy loss parameters for a standard rock shielding with $a=0.223 \mathrm{GeVmwe}$ and $b=4.64 \times 10^{-4}$ mwe as reported in Ref. [8]. However, the probability that an MCP reaches the detector with a final energy $E_{f}$ will depend not only on the penetration depth, but also on the amount of overburden that the particle must traverse. The expected overburden can be estimated by considering the incoming direction of the particle and the detector depth, such that particles arriving at detector from below are subject to a much bigger amount of overburden compared to the ones coming from above. A convolution of this probability with the flux at the surface gives a prediction 
for the flux at detector. The flux attenuation is roughly at the ten percent level for MCPs with a large $\varepsilon^{2} \gtrsim 10^{-2}$, arriving from the vertical direction with $\cos \theta=1.0$, while the upward-going flux with directions in the range $-1 \leq \cos \theta \leq 0$, is highly attenuated. For smaller $\varepsilon^{2} \lesssim 10^{-4}$, as energy losses are suppressed by two orders of magnitude, the MCP flux is mostly independent of $\cos \theta$.

\section{Millicharged particles at underground experiments}

Via the same coupling $\varepsilon$ that allows for the production of MCP in the atmosphere, the particles that reach the detector are capable of scattering off electrons in the medium via a $t$-channel photon exchange. This scattering is mediated by a massless particle, with a differential cross section that peaks for small electron recoil energy, impliying that detectors with capabilities of reconstructing low-energy electrons can exploit this. The differential cross-section is [9]

$$
\frac{d \sigma}{d E_{r}}=\varepsilon^{2} \alpha_{E M}^{2} \pi \frac{\left.m_{e}\left(E_{r}^{2}+2 E_{\chi}^{2}\right)-E_{r}\left(m_{e}\left(2 E_{\chi}+m_{e}\right)+m_{\chi}^{2}\right)\right)}{E_{r}^{2} m_{e}^{2}\left(E_{\chi}^{2}-m_{\chi}^{2}\right)},
$$

where $E_{r}$ is the electron recoil energy, $\alpha_{E M}$ is the electromagnetic coupling constant, and $m_{\chi}$ and $m_{e}$ are the masses of the MCP and the electron, respectively. For a single-scattering analysis, where the $\mathrm{MCP}$ is able to provide enough energy to an electron to leave a distinct signature in the experiment, we estimate the number of signal events in a reconstructed electron recoil energy bin $j$, by

$$
\mu_{j}^{s}=T n_{e} \int_{E_{j}-E_{b} / 2}^{E_{j}+E_{b} / 2} d E_{r} \epsilon\left(E_{j}\right) \int d \cos \theta d E_{\chi} \frac{d \Phi_{\chi}}{d E_{\chi} d \cos \theta} \frac{d \sigma}{d E_{r}}
$$

where $n_{e}$ is the total number of electrons in the detector, $T$ is the time exposure, $E_{b}$ is the bin size, $E_{j}$ is the bin center, and $\epsilon\left(E_{j}\right)$ is the average detection efficiency in a given bin $j$.

To perform this search we used the event selection from Super-Kamiokande (SK) in reference [10], and for XENON1T in [11], as well as the simulated background expected in JUNO [12]. We performed a bin-by-bin likelihood analysis and obtain constraints at $90 \%$ confidence level in the plane of the squared of charge fraction $\varepsilon^{2}$ and the MCP mass $m_{\chi}$. The results can be seen in the left panel of figure 2, where the strongest limit for $100 \mathrm{MeV} \lesssim \mathrm{m}_{\chi} \lesssim 500 \mathrm{MeV}$ is from SuperKamiokande. However, for a similar running time in JUNO (orange line) it is expected to improve over the limits of SK, due to JUNO's ability to reach very low electron recoil energy. Although XENON1T (grey) can reach keV-scale electron recoils in this analysis, its small volume-exposure limits its capabilities relative to SK or JUNO.

Another kind of signal that can be searched for, is the multiple energy deposition of MCP as it traverses the detector. If these multiple-scattering signals take place in a sufficiently small time window, then it is possible to suppres the low-energy background which increases at these energies. In this case, the signal will scale with even more powers of the charge fraction than in single-scattering analysis, as the propability of having two or more hits in the detector is

$$
P_{n \geq 2}=1-e^{-\frac{L_{\text {det. }}}{\lambda_{\mathrm{S}}}}\left(1+\frac{L_{\mathrm{det}}}{\lambda}\right)
$$

where $L_{\text {det. }}$ is the detector lenght and $\lambda$ is the mean free path, which dependes on the electron density and scattering cross-section. We performed a multiple-hit analysis in JUNO, as it is a large detector 

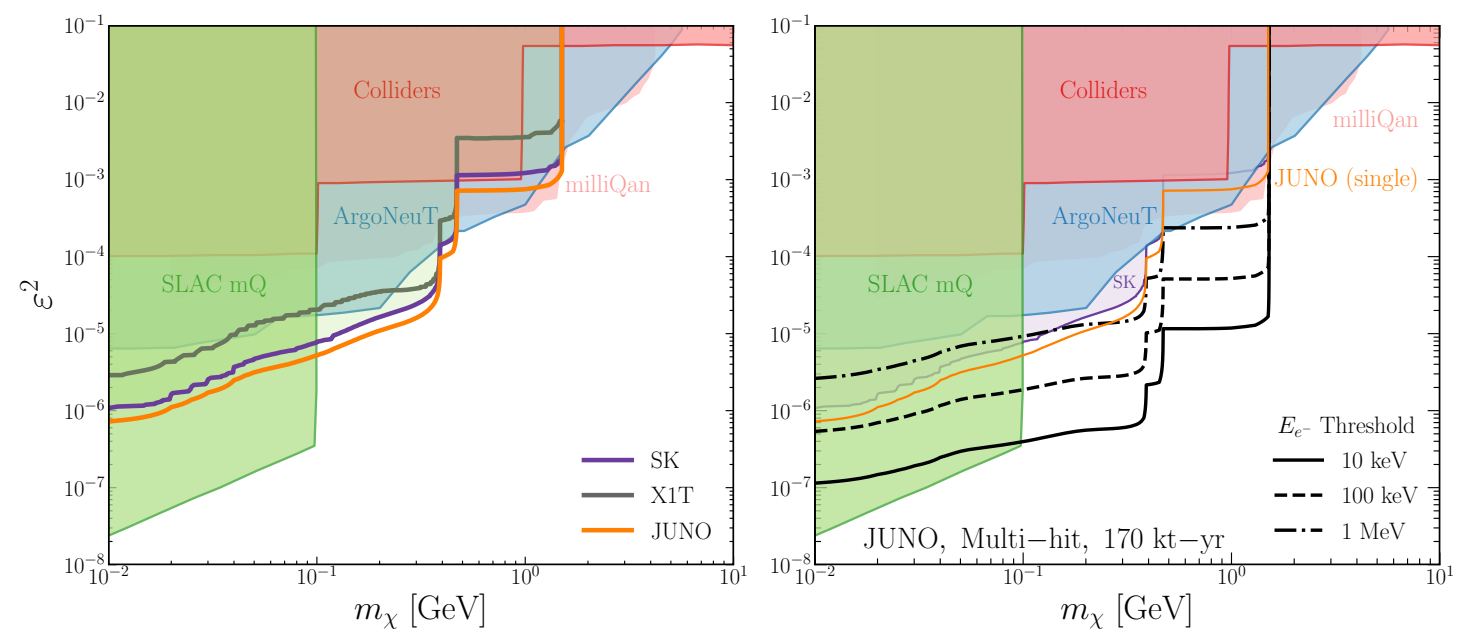

Figure 2: Left: Constraints on millicharged particle parameter space from SLAC mQ [13], ArgoNeuT [14], the milliQan Demonstrator [15], and colliders [16], compared with our 90\% CL single-hit constraints from Super-Kamiokande (purple), JUNO (orange), and XENON1T (grey). Right: Expected 90\% CL sensitivity to millicharged parameter space using the multiple hit strategy of JUNO and a $170 \mathrm{kt}-\mathrm{yr}$ exposure. Three different assumptions about the minimum observable electron recoil energy are assumed: $10 \mathrm{keV}$ (solid), $100 \mathrm{keV}$ (dashed), and $1 \mathrm{MeV}$ (dot-dashed). Existing constraints (including our SK analysis in purple) are shown as filled in regions, and also the single-hit analysis result for JUNO.

with a low energy threshold that allow us to fully exploit the advantages of the MCP signal. The right panel of figure 2 shows the results of this search where we take three different assumptions about the minimum threshold energy of electrons that JUNO can detect $-1 \mathrm{MeV}$ (dot-dashed), $100 \mathrm{keV}$ (dashed), and $10 \mathrm{keV}$ (solid). We observe that the multiple-hit strategy will far exceed single-scattering searches for atmospheric MCP.

\section{Summary}

If a particle with a small electric charge exist in nature, then it should be produced in cosmic-ray air showers. We performed a simulation of the atmospheric flux of MCPs produced in the decay of mesons in the shower. We accounted for the attenuation of the flux as the millicharged particle propagates through the earth, and derive constraints from the Super-Kamiokande and XENON1T experiments, using electron recoil data. The best current limits for MCPs with masses in the $100 \mathrm{MeV} \lesssim \mathrm{m}_{\chi} \lesssim 500 \mathrm{MeV}$ range are obtained from SK. Moreover, we estimated the capabilities of improving over these constraints in a future multi-tonne neutrino detector with a low energy threshold such as JUNO. We demonstrated that multiple-scattering signals offer a powerful means to search for MCPs, complementary to current and upcoming collider and fixed-target based searches.

Acknowledgements. This work was supported by the Faculty of Arts and Sciences of Harvard University, the Alfred P. Sloan Foundation, the Fermi Research Alliance, LLC, under contract 
DE-AC02-07CH11359 with the U.S. Department of Energy, and by ANID-PCHA/DOCTORADO BECAS CHILE/2018-72180000.

\section{References}

[1] C. D. Anderson, The Positive Electron, Phys. Rev. 43 (1933) 491-494.

[2] S. Neddermeyer and C. Anderson, Note on the Nature of Cosmic Ray Particles, Phys. Rev. 51 (1937) 884-886.

[3] M. Dobroliubov and A. Ignatiev, MILLICHARGED PARTICLES, Phys. Rev. Lett. 65 (1990) 679-682.

[4] A. Berlin, D. Hooper, G. Krnjaic and S. D. McDermott, Severely Constraining Dark Matter Interpretations of the 21-cm Anomaly, Phys. Rev. Lett. 121 (2018) 011102, [1803.02804].

[5] C. A. Argüelles Delgado, K. J. Kelly and V. Muñoz Albornoz, Millicharged Particles from the Heavens: Single- and Multiple-Scattering Signatures, 2104.13924.

[6] A. Fedynitch, R. Engel, T. K. Gaisser, F. Riehn and T. Stanev, Calculation of conventional and prompt lepton fluxes at very high energy, EPJ Web Conf. 99 (2015) 08001, [1503.00544].

[7] C. Argüelles, P. Coloma, P. Hernández and V. Muñoz, Searches for Atmospheric Long-Lived Particles, JHEP 02 (2020) 190, [1910 . 12839].

[8] J. H. Koehne, K. Frantzen, M. Schmitz, T. Fuchs, W. Rhode, D. Chirkin et al., PROPOSAL: A tool for propagation of charged leptons, Comput. Phys. Commun. 184 (2013) 2070-2090.

[9] R. Harnik, Z. Liu and O. Palamara, Millicharged particles in liquid argon neutrino experiments, Journal of High Energy Physics 2019 (Jul, 2019) .

[10] Super-Kamiokande collaboration, K. Bays et al., Supernova Relic Neutrino Search at Super-Kamiokande, Phys. Rev. D 85 (2012) 052007, [1111 . 5031].

[11] XENON collaboration, E. Aprile et al., Excess electronic recoil events in XENONIT, Phys. Rev. D 102 (2020) 072004, [2006.09721].

[12] JUNO collaboration, F. An et al., Neutrino Physics with JUNO, J. Phys. G 43 (2016) 030401, [1507.05613].

[13] A. A. Prinz, The Search for millicharged particles at SLAC, other thesis, 3, 2001.

[14] ArgoNeuT collaboration, R. Acciarri et al., Improved Limits on Millicharged Particles Using the ArgoNeuT Experiment at Fermilab, Phys. Rev. Lett. 124 (2020) 131801, [1911.07996].

[15] MILliQAn collaboration, A. Ball et al., Sensitivity to millicharged particles in future proton-proton collisions at the LHC, 2104.07151.

[16] S. Davidson, S. Hannestad and G. Raffelt, Updated bounds on millicharged particles, JHEP 05 (2000) 003, [hep-ph/0001179]. 